\title{
La Economía Social en el emprendimiento de base tecnológica en España. Un análisis cualitativo
}

\author{
Ma Isabel Martínez Martín \\ Nuria Guilló Rodríguez \\ Rosa Santero Sánchez
}

RESUMEN: Hay plena coincidencia sobre la importancia de las transformaciones sociales y económicas que las Tecnologías de la Información y la Comunicación han originado en las últimas décadas. Las empresas de base tecnológica (EBT) muestran una capacidad extraordinaria para ofrecer nuevas soluciones, tanto a las necesidades emergentes como a otras que han estado insatisfechas por largos períodos de tiempo, y su impacto económico y social es indudable por su aportación al crecimiento económico y por ser elementos indispensables en la transferencia de tecnología desde los sistemas de innovación a los mercados y la sociedad.

En este contexto cabe entender el interés de la Economía Social por tener una participación creciente en las actividades de base tecnológica o basadas en el conocimiento. Dicha participación fortalecería el sector, elevando sus tasas de crecimiento a medio y largo plazo; mejorando su contribución relativa a las macromagnitudes autonómicas y estatales; incorporando nuevos perfiles de trabajadores/as y de empresas, y elevando las condiciones laborales medias del sector. Este artículo profundiza en la relación entre las empresas de Economía Social y el emprendimiento de base tecnológica a través de un análisis cualitativo que incorpora la visión de los principales actores. A partir de ese análisis se identifican las principales líneas de avance del sector agrupadas en cuatro ejes: mejora de la visibilidad de la Economía social, acercamiento de la Economía social a los nuevos perfiles de emprendedores de base tecnológica, asesoramiento y acompañamiento en las etapas iniciales, y apoyo en las fases de crecimiento y consolidación.

PALABRAS CLAVE: Economía Social, emprendimiento de base tecnológica, emprendimiento basado en el conocimiento, sectores emergentes, análisis cualitativo, tendencias.

CLAVES ECONLIT: L31, 031, 033, M15.

Cómo citar este artículo / How to cite this article: MARTíNEZ, M.I., GUILLÓ, N. \& SANTERO, R. (2019): "La

Economía Social en el emprendimiento de base tecnológica en España. Un análisis cualitativo", CIRIEC-

España, Revista de Economía Pública, Social y Cooperativa, 96, 65-90. DOI: 10.7203/CIRIEC-E.96.12969.

Correspondencia: $\mathrm{M}^{\mathrm{a}}$ Isabel Martínez Martín, Abay Analistas Económicos, e-mail: mmartinez@ abayanalistas.net;

Nuria Guilló Rodríguez, Abay Analistas Económicos, nguillo@abayanalistas.net, y Rosa Santero Sánchez,

Universidad Rey Juan Carlos, Facultad de Ciencias Jurídicas y Sociales, rosa.santero@ urjc.es. 


\section{EXPANDED ABSTRACT}

\section{The Social Economy within Technology Based Entrepreneurship in Spain. A qualitative approach}

There is a wide consensus regarding the importance of social and economic transformations that new technologies, especially Information and Communication Technologies (ICT), have had in the last decades. ICT companies show an extraordinary capacity to offer new solutions to emerging and unsatisfied needs. Their social and economic impact is unquestionable in relation to their contribution to economic growth and they are also essential elements in the transfer of technology from national innovation systems to markets and society in general.

In this context, we can understand the interest Social Economy firms have in increasing their presence within emerging technology-based activities. This presence will contribute to strengthening the Social Economy (SE) sector in several aspects: increasing its growth rates in the medium and long term; improving its relative contribution to regional and national macro-economic indicators; incorporating new profiles of workers and companies; and improving working conditions in the sector.

\section{Objectives}

The objective of this paper is to assess the current position of SE companies in technology-based sectors and, more specifically, technology-based entrepreneurship (TBE) in the SE. In addition, it aims to delve into the different elements that condition this position, with the ultimate goal of identifying the areas of progress to improve its position in the TBE sector.

\section{Methodologic approach}

In order to achieve the goal, we chose to undertake qualitative research of a phenomenological nature (Golafshani, 2003), using in-depth interviews with a sample of actors within the main groups involved in technology-based entrepreneurship and in the SE. The following profiles were selected:

- Business and tecnological entrepreneurship advisory agents (chambers of commerce, business incubators, spin-offs);

- support structures of the SE, as representative entities and specialists, aware of the reality of the sector and its presence and position in technological sectors;

- technology-based companies and entrepreneurs, as the main players and experts with their firsthand experience in these types of business initiatives; 


\section{LA ECONOMÍA SOCIAL EN EL EMPRENDIMIENTO DE BASE TECNOLÓGICA EN ESPAÑA. UN ANÁLISIS CUALITATIVO}

- venture capital funds, as the main investors and financial agents in these types of companies at their growth and expansion stages.

Finally, twenty-two entities and individuals were selected for the interviews, which were carried out in November and December 2015 and had an approximate average duration of one hour each.

The selected methodological approach was developed through in-depth interviews (Taylor and Bogdan, 1992). This information collection technique is ideal for the proposed objectives as they allow access to information in a direct and structured way and, at the same time, facilitate the creation of analytical processes.

\section{Results}

The results obtained from the content analysis of the interviews are grouped into three categories:

\section{The importance of technology-based entrepreneurship in the Social Economy}

Emerging technology-based activities have a strategic role for the whole economy and, in particular, for the SE sector. First, these activities allow for a greater sectorial and geographical diversification. Second, technology-based activities are found in sectors with high and continuous growth in addition to high added value, thus strengthening firms. These activities also favor the incorporation of new worker profiles (younger and highly qualified), which are associated with stable jobs and high remuneration, that lead to an improvement in the average working conditions of the SE.

\section{Differential elements of technology-based entrepreneurship}

The characterization of technology-based entrepreneurship allows the differential elements of this type of entrepreneurship to be known. As such, it is possible to identifying those characteristics that pose particular difficulties for the SE entities due to their identifying elements.

For example, TBE are companies in which cooperation, the creation of networks and the generation of synergies with other companies and agents (from their own sector or from others) are key elements. They usually have an ongoing relationship with universities, research centers and technology centers. In addition, these firms tend to develop in different activity branches with high technological content and increased tendency to innovate. These companies usually present a very long maturity phase for their products, with high and rapid growth in their consolidation phases. They also have specific financial needs (seed capital and public aid during the initial phase, and venture capital in later phases). 
The advice received by these companies is usually focused on the development of the business model and the choice of the legal form for the firm is secondary. Therefore, they do not search for the advantages of the SE legal form.

3. Strategic areas to improve the situation of the Social Economy in technological -based activities or sectors.

Strategic initiatives are grouped around four specific points: improving the visibility of the SE, in particular, aimed at potential entrepreneurs and universities, research centers and technology centers, where technology-based initiatives arise; bringing the SE closer to new entrepreneurship and BTE profiles, increasingly similar to the concept of social enterprise (with more democratic and participative models in addition to a concern for a greater " social return"); counselling and support in the initial stages of BTE, in business and legal training; and support in mature and consolidation phases, especially in two areas, financial and network services.

\section{Conclusions}

Beyond the core ICT activities analysed in this paper, it is worth asking what role the Spanish Social Economy can play in the development of emerging sectors. Most of them are the result of the merger between ICTs and different activities, which of their own elements can offer them an advantage and what challenges they will need to face in order to take advantage of their potential.

Firstly, the Spanish Social Economy presents a series of characteristics that give it an advantage in the anticipated development of new activities. One of these elements is the strong productive specialization that the SE and non-profit entities have in many of the emerging activities. The SE companies (including associations, foundations and other non-profit entities) represent $8.9 \%$ of the total number of Spanish companies (DIRCE, 2017). However, in activities related to care services, their presence is particularly high: $50 \%$ of social service companies without accommodation and $37.1 \%$ of services in residential establishments. Their presence is also very significant in cultural activities (46.8\%), in sports and recreational activities (28.0\%) and partly within green economy activities.

A second element that provides the SE with an advantage is that social and organizational innovation, two of the key processes in the new scenario, are identifying elements of the SE. Social innovation is related to inclusive growth, a cross-cutting element in the development of many SE entities for decades (insertion companies, sheltered employment centers, social initiative cooperatives ...). Organizational innovation, based on the Oslo Manual, entails for example, the introduction of new business practices, new methods for a better distribution of responsibilities and decision-making. These are core elements of SE entities. 


\section{LA ECONOMÍA SOCIAL EN EL EMPRENDIMIENTO DE BASE TECNOLÓGICA EN ESPAÑA. UN ANÁLISIS CUALITATIVO}

In fact, the SE organization models could accommodate the preferences of current workers, who demand more participatory, more horizontal, more inclusive and more flexible work organization models. These environments are also more suitable for the development of social innovation initiatives.

Finally, another element of the SE is its economic and social contribution recognized at a political, social and empirical level. At a political level, the mission of SE companies is completely aligned with the objectives of the Europe 2020 Strategy and the EU Agenda for growth and employment.

However, their potential advantage as indicated in previous paragraphs may be hindered by not assuming certain challenges. One of these is the progress towards a greater digitization of the sector, which must consider the entire value chain (from the provision of services to the continuous relationship with the user). The risk of not addressing the digitalization process in a decisive way may be to lose the current leadership position that it has in several sectors (education, health services, social services, agri-food, etc.).

A second challenge focuses on the field of prospecting new products and solutions, also in accordance with social innovation. One of the strategic lines of the sector could be to address prospecting and experimentation systems for new products and solutions using digitization. A strategic foresight center and the development of new tools, such as "living labs", could play a catalytic role in the incorporation of social innovation into the sector.

KEYWORDS: Social economy, technology-based entrepreneurship, innovation, qualitative analysis, trends. 


\section{Introducción'}

Desde finales del siglo XX, el panorama económico y social ha experimentado una importante y rápida transformación producida, fundamentalmente, por la globalización y la digitalización e incorporación de las nuevas tecnologías (Sánchez-Archidona, 2017). Estos procesos transforman las estructuras productivas, dando mayor peso a aquellos sectores que alimentan la transformación digital del conjunto del tejido productivo, y que, a su vez, actúan como catalizadores de profundos cambios económicos y sociales.

Dado su potencial económico y su capacidad transformadora, es fácil comprender el interés que gobiernos y otros actores económicos tienen en el desarrollo de las TIC y de los sectores y actividades directamente vinculados a ellas, así como del emprendimiento de base tecnológica. En relación con este último, cabe señalar que una buena comprensión del mismo requiere un análisis holístico y la valoración tanto del perfil de los/as emprendedores como de las dificultades que encuentran en la puesta en marcha y consolidación de las iniciativas empresariales de base tecnológica. Además, hay que tener presente que, para que estas empresas generen no solo empleo e ingresos sino también innovación, es necesaria la confluencia de intereses entre Estado, universidades y centros tecnológicos, empresas y sociedad (Salinas y Osorio, 2012).

Tanto a nivel internacional como estatal, la delimitación más tradicional del sector está muy centrada en los servicios de informática y telecomunicaciones, aunque el auge de las TIC provoca un efecto "spillover" que inunda otras ramas de actividad tradicionales, difumina la delimitación inicial del sector TIC y plantea una continua revisión de la misma. La importancia de este fenómeno ha motivado la ampliación del ámbito de análisis de este estudio, pasando del puro sector TIC al concepto de empresa y emprendimiento de base tecnológica (Coduras, 2009, Comisión Europea, 2002, Storey y Tether, 1998). Estas actividades comparten algunas características entre las que cabe destacar: su alto potencial de creación de empleo, especialmente joven y de alta cualificación; su alto contenido en conocimiento; y la importancia del espíritu emprendedor e innovador en su desarrollo (Coduras, 2009, Escuela de Organización Industrial, 2010).

En las próximas décadas, la Unión Europea y España, en particular, deberán afrontar importantes retos a nivel económico y social; y entre los factores transformadores más decisivos del escenario socioeconómico están el cambio tecnológico y la innovación (incluida la innovación social). En este

\footnotetext{
1.- Esta contribución se apoya parcialmente en una investigación más amplia realizada por Abay Analistas y financiada por la Confederación Empresarial Española de Economía Social (CEPES). Disponible el Informe completo en:

https://docs.google.com/viewerng/viewer?url=https://www.cepes.es/files/publicaciones/92.pdf
} 


\section{LA ECONOMÍA SOCIAL EN EL EMPRENDIMIENTO DE BASE TECNOLÓGICA EN ESPAÑA. UN ANÁLISIS CUALITATIVO}

contexto, las empresas de Economía Social (ES, en adelante) ${ }^{2}$ disponen de importantes oportunidades en las actividades emergentes, puesto que se han afianzado como una forma organizativa empresarial cuyos valores identitarios fortalecen el empleo y la actividad en entornos sociales y económicos adversos (Chaves y Monzón, 2012, Sánchez-Archidona, 2017). Una mayor participación de la ES en las actividades tecnológicas contribuiría a fortalecer el sector, elevando sus tasas de crecimiento a medio y largo plazo, mejorando su contribución a las macromagnitudes autonómicas y estatales, incorporando nuevos perfiles de trabajadores/as y de empresas y elevando sus condiciones laborales medias.

Así, el objetivo de este trabajo es valorar la posición actual de las empresas de ES en los sectores de base tecnológica y, más concretamente, el emprendimiento de base tecnológica mediante fórmulas de ES, así como profundizar en los distintos elementos que condicionan dicha posición, con el fin último de identificar líneas de avance. Para ello, se plantea una investigación cualitativa de carácter fenomenológico a través de entrevistas en profundidad a una muestra de actores dentro de los principales grupos intervinientes en el emprendimiento de base tecnológica y en la ES.

El artículo se estructura en torno a cinco epígrafes, incluido este introductorio. A continuación, se presenta una revisión de la literatura sobre el emprendimiento de base tecnológica y las empresas de ES. En el tercer punto se detalla la metodología utilizada y la delimitación de los sectores de base tecnológica en los que se centra esta investigación. En el cuarto se presentan los resultados del análisis cualitativo y finalmente se concluye con una discusión y con las potenciales líneas abiertas de trabajo a futuro.

\section{Revisión de la litteratura}

El impacto de la digitalización en las empresas y entidades de ES es un tema de gran interés para el sector ${ }^{3}$. Sin embargo, este artículo no aborda los niveles ni el impacto de la digitalización de la ES, sino que trata de profundizar en los obstáculos que pueden explicar la reducida participación que actual-

2.- La Ley 5/2011 de Economía Social define ésta como: el "conjunto de actividades económicas y empresariales, que en el ámbito privado llevan a cabo aquellas entidades que (...) persiguen bien el interés colectivo de sus integrantes, bien el interés general económico o social, o ambos (...). Estas entidades se rigen por algunos principios orientadores entre los que cabe destacar la primacía de las personas y del fin social sobre el capital; la aplicación de los resultados obtenidos de la actividad económica; la promoción de la solidaridad interna y con la sociedad que favorezca el compromiso con el desarrollo local, la igualdad de oportunidades, la cohesión social, la inserción de personas en riesgo de exclusión social, la generación de empleo estable y de calidad y la sostenibilidad; y la independencia respecto a los poderes públicos".

3.- La literatura existente señala efectos positivos en su posición competitiva (Bruque et al., 2003, Del Águila y Padilla, 2010, Vargas, 2004), sus resultados empresariales (Gargallo y Pérez, 2009, Fernández y Peña, 2009), la implicación y participación de sus trabajadores/as (Bruque y Moyano, 2007), así como en el compromiso de la empresa con la innovación (Espasandín, Casanueva y Ganaza, 2004). 
mente tiene en la oferta de servicios vinculados directamente a las tecnologías de la información y la comunicación.

Las oportunidades y retos que las transformaciones sociales tienen para la ES, así como la importante contribución que ésta puede realizar a los retos sociales a los que nos enfrentamos es un tema recurrente en la literatura (Cuadrado y Ciruela, 2015, Martínez et al., 2013; Martínez, Guilló, Santero y Castro, 2017, Sánchez y Pérez, 2015). Una vía principal para articular esta aportación es el emprendimiento. Algunos autores han analizado el papel importante de las políticas públicas en el apoyo a estas iniciativas emprendedoras y concluyen la necesidad de una mayor implicación y coordinación en el conjunto de actores afectados (Salinas y Osorio, 2012, Martínez, Guilló y Santero, 2016). Estos trabajos han señalado la importancia que para la ES tiene el emprendimiento en los denominados sectores emergentes, muchos de ellos procedentes de una acelerada incorporación de tecnología a actividades en las que la ES ya tiene una posición competitiva muy sólida (economía verde, sector cultural, economía azul, sector de servicios sanitarios y cuidados...). Sin embargo, la tecnología es un elemento central en las transformaciones productivas actuales y futuras y, por tanto, cabe señalar, por razones de posicionamiento estratégico, el interés de una mayor participación de la ES en las actividades nucleares de la revolución tecnológica actual, es decir, en el emprendimiento de base tecnológica.

El emprendimiento que nace de la tecnología, o el concepto de empresa de base tecnológica (EBT, en adelante) ha evolucionado en el tiempo, aunque mantiene dos características comunes en las diferentes definiciones (véase Trenado y Huergo, 2007, para mayor detalle). La primera de ellas es que la empresa debe ser de propiedad independiente y, la segunda, que su actividad esté basada en la explotación de una innovación tecnológica. En la literatura también se acepta como EBT, a las empresas que se establecen en un sector de alta tecnología o con un índice de recursos tecnológicos elevado. Más recientemente, el término se ha sustituido por el de "empresas basadas en el conocimiento" para ampliar su ámbito de referencia y dar cabida al conjunto de spin-offs creadas por empresas 0 grupos de investigadores/as para comercializar los resultados de su producción científica, sea ésta en el ámbito tecnológico o en otro (Vargas, 2012).

El sector de las TIC parece tener un peso reducido en el emprendimiento realizado desde la ES. Así, en el análisis del perfil de los/as emprendedores que han formalizado sus iniciativas empresariales a través de cooperativas y sociedades laborales en la región de Andalucía, llevado a cabo por Cuadrado y Ciruela (2015), el sector de las nuevas tecnologías y comunicaciones representa solo el $7,8 \%$, aunque con diferencias provinciales importantes, destacando el caso de Málaga con un 21,4\%.

La literatura dedicada a la ES y el emprendimiento de base tecnológica o basado en el conocimiento en España es muy escasa. Un trabajo pionero para España en la cuantificación y posición que tiene la ES en los sectores emergentes de base tecnológica, término que se corresponderían con un concepto amplio de EBT y que incluye empresas de actividades con un índice de recursos tecnológicos elevado, se encuentra en Martínez, Guilló y Santero (2016). Este trabajo, que se apoya en la 


\section{LA ECONOMÍA SOCIAL EN EL EMPRENDIMIENTO DE BASE TECNOLÓGICA EN ESPAÑA. UN ANÁLISIS CUALITATIVO}

Muestra Continua de Vidas Laborales y está referido al año 2015, confirma la menor presencia relativa de la ES en las actividades nucleares de sectores TIC y las mejores condiciones laborales de las mismas.

Las autoras aportan algunos resultados de interés para contextualizar la situación actual de la ES en el emprendimiento de base tecnológica. En términos de empleo, los sectores de base tecnológica representan el 5,6\% de la economía española, y las Industrias creativas y las Industrias de servicios móviles son los subsectores de mayor tamaño (2,8\% y el 2,5\% respectivamente). Los puestos de trabajo de estas actividades muestran algunos elementos diferenciales, como la reducida presencia de mujeres, su menor edad media, su alta cualificación y sus mejores condiciones laborales, que se concretan en un mayor porcentaje de empleados/as a tiempo completo, mayor estabilidad y salarios medios más elevados.

El mismo trabajo cuantifica la presencia de las empresas y entidades 4 de la ES en el empleo total de estas actividades, que alcanza el 6,1\%. No obstante, existen notables diferencias por comunidades autónomas (por ejemplo, en el País Vasco, este porcentaje aumenta hasta el 8,5\%) y por subsectores (en las Industrias de experiencias la economía social alcanza el $26 \%$ del empleo total frente a menos del $1 \%$ en las Industrias de servicios móviles). Este trabajo confirma, así, la escasa participación de la ES en las actividades nucleares de las TIC. Un análisis comparado de las condiciones laborales que se observan en las empresas de la ES frente a un grupo de control de empresas mercantiles confirma que también en estos sectores se observan mayores niveles de equidad, tal como sucede en el conjunto del tejido productivo (Castro, Santero, Martínez y Guilló, 2013).

Desde una perspectiva jurídico administrativa, Vargas (2012) analiza las limitaciones que las empresas de ES (en concreto, cooperativas y sociedades laborales) tienen para ser EBT nacidas en el ámbito académico. El autor identifica barreras legales que dificultan la elección de estas empresas a la hora de constituir una spin-off académica. La más significativa es el hecho, altamente probable, de que los profesores o investigadores trabajen a tiempo completo y en régimen de exclusividad en la universidad, lo que no les permite realizar una actividad laboral continuada en otra empresa o entidad de carácter privado. Y este último aspecto es un elemento clave en las empresas de ES. Además, la participación de la universidad en el capital de la empresa, tendencia también creciente, es más fácil en las empresas capitalistas.

Seguí-Mas, Sarrión-Viñes, Tormo-Carbó y Oltra (2013) realizan una búsqueda sistemática de spinoff creadas bajo fórmulas de cooperativa en las universidades españolas y constatan que esta forma jurídica es muy escasa si se compara con el resto de formas jurídicas, confirmando así los resultados de otras investigaciones previas (Ortín et al., 2007). Seguí-Mas et al. (2013) identifican una población de ocho spin-off cooperativas, y realizan 6 estudios de casos. Los resultados de su análisis

4.- Para mayor información sobre el tipo de entidades incluidas y la clasificación de los sectores emergentes de base tecnológica, véase el capítulo 2 del informe. 
destacan que la principal motivación para elegir esta forma jurídica es la apuesta por fórmulas de empleo horizontales y de participación democrática, junto con la independencia y el deseo de lograr avances en el conocimiento tecnológico. Entre las principales ventajas de elegir esta forma societaria, los emprendedores señalan las fiscales y las vinculadas a los recursos humanos (la implicación de los socios en el proyecto) y, entre las principales dificultades, las financieras y las relacionadas también con los recursos humanos. Entre los recursos específicos que en mayor medida ayudan a estas empresas, se señalan la disponibilidad de una incubadora para alojar el proyecto y los recursos financieros para las primeras etapas.

\section{Aproximación metodológica}

\subsection{Metodología de análisis}

El objetivo de este trabajo es profundizar en una de las potenciales causas de la escasa presencia de la ES en los sectores de base tecnológica, que es su reducida participación en el emprendimiento tecnológico y, más concretamente, valorar la situación actual, determinar los elementos que condicionan su posicionamiento, e identificar líneas de avance para elevar esta participación.

Para alcanzar este objetivo se plantea una investigación cualitativa con diseño fenomenológico que trata de comprender un fenómeno en un contexto específico (Golafshani, 2003), en nuestro caso, el emprendimiento de base tecnológica a través de fórmulas de la ES, desde una perspectiva amplia de diferentes actores sociales participantes. La selección de esta metodología permite acercarse al objetivo de la investigación, y profundizar en los principales elementos favorecedores u obstaculizadores que detectan los actores, sin tratar de manipular el fenómeno, sino con la finalidad de entenderlo.

Esta aproximación metodológica seleccionada se desarrolla a través de entrevistas en profundidad (Taylor y Bogdan, 1992). Esta técnica de recogida de información resulta idónea para los objetivos planteados ya que permite el acceso a la misma de una forma directa y estructurada y, al mismo tiempo, facilita la generación de procesos de reflexión.

Para facilitar la categorización de la información y su posterior análisis, se diseñó un guion "ad hoc" que permitió centrar el contenido de las entrevistas realizadas y tratar los temas claves para la investigación. Este guión fue facilitado previamente a los actores a entrevistar y, aunque la realización de las entrevistas se planteó de forma abierta con el fin de acceder a la información desde la pers- 


\section{LA ECONOMÍA SOCIAL EN EL EMPRENDIMIENTO DE BASE TECNOLÓGICA EN ESPAÑA. UN ANÁLISIS CUALITATIVO}

pectiva de cada uno de los actores involucrados, durante las entrevistas siempre se mantuvo el foco en aquellos aspectos determinados como clave por el equipo investigador.

En base a estas premisas, y teniendo en cuenta que el objetivo último de la investigación radicaba en valorar la posición de la ES en los sectores emergentes de base tecnológica y profundizar, de manera específica, en la relación entre la ES y el emprendimiento de base tecnológica, así como en los distintos elementos que actualmente condicionan su posicionamiento, se realizó una selección de actores que pudieran responder a estas cuestiones eligiendo los siguientes perfiles:

- agentes de asesoramiento empresarial (Cámaras de comercio, empresas especializadas en asesoría y gestoría) y de asesoramiento ligado al emprendimiento tecnológico (viveros de empresa, spin-offs, incubadoras y aceleradoras);

- estructuras de apoyo de la ES, como entidades representativas y especialistas, conocedoras de la realidad del sector y de su presencia y posición en el emprendimiento de base tecnológica;

- empresas y emprendedores/as de base tecnológica, en su papel de protagonistas y narradores de su experiencia en primera persona en este tipo de iniciativas empresariales; $y$

- fondos de capital riesgo, como inversores principales en este tipo de empresas y financiadores clave de sus etapas de crecimiento y expansión.

Finalmente, se seleccionaron veintidós entidades y personas para las entrevistas, que se llevaron a cabo en los meses de noviembre y diciembre de 2015 y cada una de ellas tuvo una duración media aproximada de una hora. El contacto con las entidades, la gestión y las entrevistas fueron realizados por personal técnico experto en investigación cualitativa, grabando en audio estas últimas. El listado de actores entrevistados se presenta en la Tabla 1, detallando en cada caso el perfil de cada uno de ellos y el motivo por el cual se realizó su selección 5 .

A partir de las entrevistas, se ha procedido a hacer un análisis de contenido (Rodríguez Gómez, Gil Flores y García Jiménez, 1996), como herramienta metodológica para el análisis, teniendo en cuenta los aspectos más relevantes del objetivo de la investigación, y la existencia de información previa a las entrevistas (análisis cuantitativo realizado en el marco de esta misma investigación) sobre el fenómeno a estudiar que requería de una mayor profundización en la materia. Debido a la amplitud de aproximaciones que pueden emplearse en el propio análisis de contenido, que van desde los análisis más interpretativos hasta los más sistemáticos (Rosengren, 1981), se consideró conveniente centrar la aproximación metodológica en el uso del análisis dirigido de contenidos en cuanto que este permite que el proceso de investigación se realice de forma más estructurada que el análisis convencional (Hsieh y Shannon, 2005).

5.- En algún caso se entrevistó a más de una persona de la misma entidad o empresa. 


\section{Tabla 1. Entidades y expertos/as entrevistados}

\begin{tabular}{|c|c|c|}
\hline ENTIDAD-EXPERTO/A & TIPO DE ACTOR & MOTIVO SELECCIÓN \\
\hline $\begin{array}{l}\text { ANEL (Asociación de Empresas } \\
\text { de Economía Social de Navarra) }\end{array}$ & Estructura de apoyo a la ES & Grado de conocimiento de la ES \\
\hline APTENT SOLUCIONES, S.L. & EBT- economía mercantil & $\begin{array}{l}\text { Motivos, facilitadores y obstáculos al emprendimiento EBT. } \\
\text { Grado de conocimiento de la ES y sus posibilidades como EBT }\end{array}$ \\
\hline LEAN STARTUP & $\begin{array}{l}\text { Asesoramiento a emprendedo- } \\
\text { res/as y EBT }\end{array}$ & $\begin{array}{l}\text { Motivos, facilitadores y obstáculos al emprendimiento EBT. } \\
\text { Grado de conocimiento de la ES y sus posibilidades como EBT }\end{array}$ \\
\hline BITERGIUM, S.L.L. & EBT- economía social & $\begin{array}{l}\text { Motivos, facilitadores y obstáculos al emprendimiento EBT en } \\
\text { ES }\end{array}$ \\
\hline CÁMARA DE GRAN CANARIA & $\begin{array}{l}\text { Asesoramiento a emprendedo- } \\
\text { res/as }\end{array}$ & $\begin{array}{l}\text { Motivos, facilitadores y obstáculos al emprendimiento en gene- } \\
\text { ral. Grado de conocimiento de la ES y sus posibilidades como } \\
\text { EBT }\end{array}$ \\
\hline $\begin{array}{l}\text { UNIVERSIDAD POLITÉCNICA DE } \\
\text { CARTAGENA }\end{array}$ & $\begin{array}{l}\text { Universidad. Cátedra Banco del } \\
\text { Emprendimiento del Banco } \\
\text { Santander. }\end{array}$ & $\begin{array}{l}\text { Conocimiento académico y experiencia en asesoramiento } \\
\text { empresarial a emprendedores/as }\end{array}$ \\
\hline $\begin{array}{l}\text { CEIN (Centro Europeo de } \\
\text { Empresas e Innovación de } \\
\text { Navarra, S.L.) }\end{array}$ & $\begin{array}{l}\text { Vivero de innovación y acelera- } \\
\text { dora empresarial }\end{array}$ & $\begin{array}{l}\text { Motivos, facilitadores y obstáculos al emprendimiento y creci- } \\
\text { miento de las empresas EBT. Grado de conocimiento de la ES y } \\
\text { sus posibilidades como EBT }\end{array}$ \\
\hline DAD (Digital Assets Deployment) & Incubadora de empresas EBT & $\begin{array}{l}\text { Motivos, facilitadores y obstáculos al emprendimiento y creci- } \\
\text { miento de las empresas EBT. Grado de conocimiento de la ES y } \\
\text { sus posibilidades como EBT }\end{array}$ \\
\hline $\begin{array}{l}\text { ETC INVENTIONS, S.COOP. } \\
\text { MAD. }\end{array}$ & EBT- economía social & $\begin{array}{l}\text { Motivos, facilitadores y obstáculos al emprendimiento EBT en } \\
\text { ES. }\end{array}$ \\
\hline EURADIA & $\begin{array}{l}\text { Consultora de proyectos multilate- } \\
\text { rales }\end{array}$ & $\begin{array}{l}\text { Posibilidades, oportunidades, facilitadores y obstáculos de las } \\
\text { EBT en ES en relación con la financiación }\end{array}$ \\
\hline FUNDACIÓN ISIS & $\begin{array}{l}\text { Fondo de capital riesgo para ini- } \\
\text { ciativas de inversión social }\end{array}$ & $\begin{array}{l}\text { Posibilidades, oportunidades, facilitadores y obstáculos de las } \\
\text { EBT en ES en relación con la financiación }\end{array}$ \\
\hline GEKO NAVSAT, S.L. & EBT- economía mercantil & $\begin{array}{l}\text { Motivos, facilitadores y obstáculos al emprendimiento EBT. } \\
\text { Grado de conocimiento de la ES y sus posibilidades como EBT }\end{array}$ \\
\hline $\begin{array}{l}\text { GLOBAL SPORTS INNOVATION } \\
\text { CENTER POWERED BY MICRO- } \\
\text { SOFT }\end{array}$ & Incubadora start-ups EBT & $\begin{array}{l}\text { Motivos, facilitadores y obstáculos al emprendimiento y creci- } \\
\text { miento de las empresas EBT. Grado de conocimiento de la ES y } \\
\text { sus posibilidades como EBT }\end{array}$ \\
\hline GRUPO INTEGRITAS & $\begin{array}{l}\text { Empresa de servicios de asesora- } \\
\text { miento a empresas en los ámbitos } \\
\text { legal, contable, financiero y fiscal. }\end{array}$ & $\begin{array}{l}\text { Grado de conocimiento de la EBT. Posibles características dife- } \\
\text { renciales de las empresas y entidades de ES en EBT (fiscal, } \\
\text { financiero, legal, ...) }\end{array}$ \\
\hline
\end{tabular}




\begin{tabular}{|l|l|l|}
\hline ENTIDAD-EXPERTO/A & TIPO DE ACTOR & MOTIVO SELECCIÓN \\
\hline $\begin{array}{l}\text { INNOBASQUE (Agencia vasca de } \\
\text { la innovación) }\end{array}$ & Agente clave en innovación & $\begin{array}{l}\text { Motivos, facilitadores y obstáculos al emprendimiento EBT. } \\
\text { Grado de conocimiento de la ES y sus posibilidades como EBT }\end{array}$ \\
\hline PARQUE CIENTÍFICO UC3M & Vivero de empresas EBT - Spin off & $\begin{array}{l}\text { Motivos, facilitadores y obstáculos al emprendimiento EBT. } \\
\text { Grado de conocimiento de la ES y sus posibilidades como EBT }\end{array}$ \\
\hline Asesora Experta en capital riesgo & Capital riesgo & $\begin{array}{l}\text { Posibilidades, oportunidades, facilitadores y obstáculos de las } \\
\text { EBT en ES en relación con la financiación }\end{array}$ \\
\hline $\begin{array}{l}\text { SAIOLAN } \\
\text { UCOMUR (Unión de Cooperativas } \\
\text { de la Región de Murcia) }\end{array}$ & $\begin{array}{l}\text { Estructura de apoyo a la ES } \\
\text { empresarial del ámbito de la ES }\end{array}$ & $\begin{array}{l}\text { Motivos, facilitadores y obstáculos al emprendimiento EBT en } \\
\text { ES }\end{array}$ \\
\hline $\begin{array}{l}\text { Vivero de la UNIVERSIDADES DA } \\
\text { CORUÑA }\end{array}$ & Vivero de empresas EBT - Spin off & $\begin{array}{l}\text { Motivos, facilitadores y obstáculos al emprendimiento EBT. } \\
\text { Grado de conocimiento de la ES y sus posibilidades como EBT }\end{array}$ \\
\hline
\end{tabular}

FUENTE: Elaboración propia.

\section{2. Ámbito del análisis: delimitación de las actividades emergentes de base tec- nológica}

La delimitación de actividades de base tecnológica que configura el ámbito de este análisis se ajusta a la realizada por Monfardini (2012). Este, desarrolló un amplio trabajo dirigido a identificar6 y clasificar ${ }^{7}$ las "industrias emergentes", que integran actividades económicas basadas en nuevos productos, servicios, tecnologías o ideas que están en sus primeras etapas de desarrollo y que se caracterizan por sus altas tasas de crecimiento (presentes y esperadas) y la amplitud de sus mercados potenciales. Estas actividades comparten algunas características, entre las que cabe señalar dos, directamente ligadas al interés de esta investigación:

- Incluyen tanto actividades económicas completamente nuevas como procedentes de sectores reestructurados que se están transformando o que están evolucionado por las intersecciones encontradas con otras actividades. Estas últimas proceden frecuentemente de "spillovers" tecnológicos, y dan lugar a actividades híbridas.

- Son actividades intensivas en conocimiento y en investigación, que surgen frecuentemente de innovaciones disruptivas, y de la aplicación de la creatividad.

6.- En este trabajo se afirma que, en líneas generales, los sectores experimentan un ciclo de vida similar al de una empresa o un producto individual. Un sector no es más que la agregación de un amplio número de iniciativas empresariales en distintos momentos de su línea vital. $Y$, en la medida en la que estas evolucionan con una fuerte concentración en las fases de su ciclo de vida, determinan también la fase en la que se encuentra el sector.

7.- Los sectores emergentes incluyen: industrias creativas, ecoindustrias, industrias de experiencias, industrias marítimas, industrias de servicios móviles, industrias de la movilidad e industrias de la medicina personalizada. 
La clasificación de sectores emergentes de Monfardini (2012) es muy heterogénea. El ámbito de análisis de este artículo se circunscribe a un subgrupo de ellas en las que el cambio técnico es un elemento nuclear, denominados "sectores emergentes de base tecnológica", y que incluirían las Industrias de servicios móviles; las Industrias creativas, integradas por las industrias vinculadas a la generación de contenidos y a la distribución de productos TIC y un conjunto de actividades económicas, principalmente de servicios, que están siendo "colonizadas" por las TIC; y las Industrias de experiencias que ya presentan un altísimo contenido $\mathrm{TIC}$, como es el caso de la gestión de reservas turísticas a través de plataformas y portales 0 bien se espera que próximamente se vean afectadas de forma radical por ellas (servicios de creación artística, cultural y espectáculos).

\section{Posicionamiento de la Economía Social en el emprendimiento de base tecnológica. Resultados del análisis cualitativo}

Los resultados obtenidos tras el análisis de contenido de dichas entrevistas se presentan a continuación agrupados en tres bloques. El primero, incluye la visión de los actores sobre cuál es la situación actual de la ES en el emprendimiento de base tecnológica y el impacto que podría tener sobre su tejido empresarial. Las actividades emergentes de base tecnológica tienen un papel estratégico para la ES, tanto por sus características como por las implicaciones que para la misma tendría una mayor diversificación sectorial y una mayor presencia en sectores de alto crecimiento y alto valor añadido. El segundo bloque, caracteriza el emprendimiento de base tecnológica y aporta los elementos diferenciales de este emprendimiento, permitiendo identificar aquellos que son dificultades especiales para las empresas de ES por sus elementos identitarios, y que deben ser líneas prioritarias de actuación para aumentar la presencia de la ES en este ámbito, que se presentan en el último bloque.

\subsection{Importancia del emprendimiento de base tecnológica para la Economía Social}

La ES es una realidad económica y social muy importante en España ${ }^{8}$, con una presencia muy relevante en múltiples sectores de actividad, entre otros, el agroalimentario, la educación, los servicios sociales o los servicios culturales. Asimismo, la ES tiene una presencia significativa en el conjunto de las actividades emergentes de base tecnológica, pero su importancia cuantitativa desciende notablemente en las actividades nucleares de las $\mathrm{TIC}^{9}$.

8.- Se puede encontrar información actualizada sobre la Economía Social en la web de CEPES (www.cepes.es).

9.- Martínez et al. (2016): "Fomento de la presencia de la Economía Social en el emprendimiento de base tecnológica y otras actividades emergentes" Disponible en: https://docs.google.com/viewerng/viewer?url=https://www.cepes.es/files/publicaciones/92.pdf 


\section{LA ECONOMÍA SOCIAL EN EL EMPRENDIMIENTO DE BASE TECNOLÓGICA EN ESPAÑA. UN ANÁLISIS CUALITATIVO}

Atendiendo a las características de los sectores emergentes de base tecnológica, especialmente los nucleares, estos podrían tener un interés estratégico para la ES, ya que, tal como apunta la literatura especializada en la materia y, en líneas generales comparten los actores entrevistados, una mayor presencia de la misma en estas actividades contribuiría a fortalecer el sector por distintas vías.

En primer lugar, al aumentar su participación en actividades con altas tasas de crecimiento esperado sostenidas en el tiempo, en el medio y largo plazo cabe esperar que se eleve también el crecimiento medio del sector. Las actividades emergentes de base tecnológica generan un alto valor añadido bruto y, por tanto, en términos relativos, tienen una mayor contribución al PIB, por lo que ayudarían a mejorar la contribución de la ES a la economía en general.

Por otra parte, para la ES supondría una mayor diversificación sectorial, propiciando una mayor presencia de la misma en nuevos ámbitos productivos, ahora con escasa presencia, y consecuentemente, también una diversificación geográfica.

Las EBT favorecen la incorporación de nuevos perfiles de trabajadores/as, ya que las empresas tecnológicas cuentan con un alto porcentaje de empleo joven y de muy alta cualificación, lo que generaría, asimismo, perfiles de empresas innovadoras. Estos perfiles, se asocian con empleos estables y de alta retribución, lo que propiciaría también una mejora de las condiciones laborales medias de la ES.

\subsection{Elementos diferenciales del emprendimiento de base tecnológica}

Una vez determinados los potenciales beneficios que la ES obtendría al aumentar su presencia a través de EBT, en este bloque se profundiza, desde el punto de vista y conocimiento de los actores entrevistados, en los elementos distintivos del emprendimiento de base tecnológica (Cuadro 1), esto es, en el ecosistema emprendedor en el que surgen y crecen las empresas del subsector de actividades nucleares de las TIC, vinculadas estrechamente a los servicios de informática y de telecomunicaciones, entendidos ambos en sentido amplio.

Habitualmente, las EBT son empresas en las que la cooperación, la creación de redes y la generación de sinergias con otras empresas y agentes (de su mismo sector o de otros) son elementos clave. Suelen tener una relación continua con universidades, centros de investigación, centros tecnológicos y OTRl's. A menudo, son estas entidades las que ejercen como asesoras y prescriptoras cuando en el proceso inicial de la actividad empresarial, existiendo diferentes agentes ligados al ámbito tecnológico (viveros de empresa, spin-offs, incubadoras y aceleradoras) que integran los circuitos propios de este tipo de emprendimiento y cuya visión debe considerarse para entenderlo. 
En relación a la sectorización, las EBT tienden a desarrollarse en ramas de actividad diversas, pero con un nexo común, que es el alto contenido tecnológico y una elevada propensión a la innovación. Suelen además concentrarse en clústeres 0 determinadas áreas geográficas.

Son empresas formadas por equipos multidisciplinares, donde el trabajo en equipo se valora muy positivamente; $y$ los perfiles profesionales que incorporan inicialmente suelen tener vinculación con los ámbitos científicos, tecnológicos y con la l+D, con una alta cualificación y especialización.

Estas empresas suelen tener una fase de maduración de sus productos muy prolongada, con crecimientos altos y rápidos en sus fases de consolidación. Esta característica afecta directamente a las necesidades de financiación. Las empresas de base tecnológica recurren, mayoritariamente, al capital semilla y a las ayudas públicas durante su fase inicial, y al capital riesgo en fases posteriores.

\section{Cuadro 1. Elementos diferenciales de EBT}

\begin{tabular}{|c|}
\hline Cooperación, redes y clústeres \\
\hline Relación con centros de investigación \\
\hline Ramas de actividad diversas, con alto contenido tecnológico e innovador \\
\hline Equipos multidisciplinares con alta especialización y cualificación \\
\hline Falta de formación en materia empresarial y jurídica \\
\hline Necesidades especíicas de financiación (capital semilla en inicio, y capital riesgo en consolidación) \\
\hline Consideración de la forma jurídica como un elemento secundario \\
\hline
\end{tabular}

FUENTE: Elaboración propia.

Adicionalmente, existen otros elementos que pueden estar condicionando la presencia de EBT en la ES y que emergen del discurso de los actores entrevistados, fundamentalmente relacionados con la falta de interés que los/as emprendedores/as de base tecnológica suelen mostrar por la forma jurídica en la que constituyen sus empresas, y que les lleva a elegir aquellas que les resultan más familiares 0 conocidas.

Uno de los motivos es la falta de formación que estos/as emprendedores/as tienen en materia empresarial, lo que determina su desconocimiento sobre las implicaciones de constituirse en una u otra forma jurídica. En este sentido, pueden suponer que la forma jurídica en la que se emprende no tiene porqué condicionar la organización interna de la empresa o ser relevante a la hora de tomar decisiones. 


\section{LA ECONOMÍA SOCIAL EN EL EMPRENDIMIENTO DE BASE TECNOLÓGICA EN ESPAÑA. UN ANÁLISIS CUALITATIVO}

Ligado a lo anterior, el tipo de asesoramiento mayoritario que reciben estas iniciativas está más centrado en el desarrollo del modelo de negocio que en otras cuestiones como la elección de la forma jurídica del emprendimiento y sus correspondientes características legales, tributarias o laborales.

\subsection{Líneas de avance de la Economía Social en las actividades de base tecnológica}

Una vez ofrecida una breve contextualización sobre la importancia del emprendimiento de base tecnológica para la ES e identificados los principales elementos que están determinando su presencia en este tipo de actividades, este epígrafe se centra en aquellos aspectos o lugares en los que los actores entrevistados consideran que la ES puede actuar para mejorar su posicionamiento.

Las líneas de avance se recogen en cuatro puntos concretos (Cuadro 2) que se corresponden con una agrupación temática de aquellos aspectos donde se ha detectado que una actuación eficaz podría traducirse en resultados significativos para la Economía Social.

\section{Cuadro 2. Potenciales líneas de actuación en la ES para avanzar en EBT}

\begin{tabular}{|l|l|}
\hline 1. Mejorar la visibilidad de la ES & $\begin{array}{l}\text { - En el sistema educativo } \\
\text { - En el sistema de Ciencia y Tecnología } \\
\text { - En los agentes de asesoramiento empresarial }\end{array}$ \\
\hline $\begin{array}{l}\text { 2. Acercamiento de la ES a nuevos perfiles de emprendimiento, EBT con intereses próximos al concepto de empresa social } \\
\text { (modelos participativos y con retorno social) }\end{array}$ \\
\hline 3. Asesoramiento y acompañamiento en fases iniciales de EBT, en formación empresarial y jurídica especialmente \\
\hline 4. Apoyo en fases creciente y de consolidación & $\begin{array}{l}\text { - En financiación: búsqueda de financiación ajena, socios industriales y/o } \\
\text { préstamos participativos; creación de instrumentos propios } \\
\text { - En cooperación: redes y puntos de encuentro }\end{array}$ \\
\hline
\end{tabular}

FUENTE: Elaboración propia.

\subsubsection{Mejora de la visibilidad de la Economía Social}

La ES, pese a su importancia en términos económicos, creación de riqueza y contribución a la cohesión social y territorial, es aún desconocida por una gran parte de la sociedad, incluyendo también a los/as potenciales emprendedores/as y, más concretamente, a los de base tecnológica. 
En este sentido, los actores entrevistados coinciden en señalar la importancia de realizar actuaciones dirigidas a incrementar el conocimiento sobre qué es el sector, cuáles son sus principios orientadores, sus elementos específicos y sus características organizativas y, sobre todo, su importancia económica y social. Estas actuaciones, han de ir orientadas al conjunto de la sociedad, en general, pero también se han de realizar medidas específicas dirigidas a potenciales emprendedores/as. Sería conveniente que las entidades representantes y promotoras de la ES ejercieran el liderazgo en su desarrollo y realización.

En esta línea, los ámbitos en los que se han de priorizar las actuaciones de visibilización deberían ser:

- El sistema educativo en todos sus niveles, aunque prestando especial atención a aquellos donde las actuaciones podrían tener una mayor eficacia, como las áreas de conocimiento más vinculadas a las ramas de tecnología de la educación secundaria, de la Formación Profesional de grado superior y del ámbito universitario.

- El Sistema de Ciencia y Tecnología y los actores que lo conforman (Parques Tecnológicos, OTRl's, CEl's, Universidades, etc.). En líneas generales, este tipo de agentes tiene un conocimiento limitado sobre qué es la Economía Social, sus características y requisitos o las posibilidades y potenciales beneficios que sus formas jurídicas pueden aportar a las iniciativas empresariales de base tecnológica, por lo que no se ofrece un asesoramiento adecuado en la materia a los/as emprendedores/as. En este sentido, se hace necesario realizar actuaciones dirigidas a incrementar la formación a estos actores.

- Agentes de asesoramiento empresarial, ya que suelen tener una influencia decisiva en la constitución de las empresas, bien por su relación directa con el asesoramiento en temas de emprendimiento (cámaras de comercio, servicios específicos de los ayuntamientos, departamentos o concejalías de juventud), bien por ser referentes como entidades auxiliares en el proceso de constitución formal de las iniciativas (asesorías y gestorías) y cuya recomendación es determinante en la mayoría de los casos. En líneas generales, estos agentes suelen tener un conocimiento limitado sobre las formas jurídicas de la ES, por lo que suelen recomendar otras formas jurídicas que les son más conocidas o más fáciles de constituir.

\subsubsection{Acercamiento de la Economía Social a los nuevos perfiles de emprendedores/as de base tecnológica}

Desde el punto de vista de las personas entrevistadas, el escenario actual presenta ciertos elementos que pueden ejercer como impulsores de la presencia de la ES en las actividades nucleares de los sectores emergentes.

Así, algunos de los actores entrevistados apuntan a la aparición y consolidación de un nuevo perfil de emprendedor/a, con una inclinación más social, procedencia de distintos entornos y con tra- 


\section{LA ECONOMÍA SOCIAL EN EL EMPRENDIMIENTO DE BASE TECNOLÓGICA EN ESPAÑA. UN ANÁLISIS CUALITATIVO}

yectorias diversas, ligado a los cambios que está experimentando la sociedad en su conjunto y al "boom" del emprendimiento. Este nuevo perfil, liderado por gente joven, está llegando al mundo empresarial con una mentalidad "diferente", buscando crear y organizar sus empresas siguiendo modelos más democráticos y participativos y con una mayor preocupación por el "retorno social", es decir, se estaría acercando al emprendimiento con valores, principios y comportamientos muy cercanos a la ES, o al emprendedor social, que identifica una oportunidad de negocio que está basada en la resolución de un problema social, pero que plantea una solución innovadora y creativa, apoyada en el desarrollo de un proyecto empresarial (Sánchez y Pérez, 2015).

Sin embargo, esta mayor proximidad aún no se está traduciendo, de forma proporcional, en la creación de empresas en ES, probablemente relacionado, por un lado, con la ya señalada falta de interés sobre la forma jurídica en la que constituyen sus empresas y, por otro, por el limitado conocimiento que, tanto las asesorías y gestorías como los/as propios/as emprendedores/as, tienen sobre la ES, lo que conlleva que no se consideren a este tipo de empresas.

Por tanto, es necesario la visibilización del sector, sobre todo, mediante el establecimiento de referentes que contribuyan a introducir la ES en el imaginario de los/as nuevos/as emprendedores/as como una realidad en la que articular y dar coherencia a los principios sobre los que quieren organizar sus empresas. Un caso de estudio, y posible referente, tanto en la incorporación de tecnología como en la innovación en la ES, es el de Mondragón Corporación Cooperativa (Bakaikoa et al., 2014).

Al mismo tiempo, se podrían realizar actuaciones dirigidas a potenciar el acercamiento a estos perfiles, ofreciéndoles información sobre el comportamiento diferencial de las empresas de la Economía Social, resaltando su mayor resiliencia ante épocas adversas, como se ha demostrado durante la crisis, y destacando la mayor protección que proporcionan a sus integrantes, ya que estas especificidades pueden ser muy atractivas para estos nuevos tipos de emprendedores/as.

\subsubsection{Asesoramiento y acompañamiento en las fases iniciales}

La fase de constitución de cualquier empresa es un momento de especial dificultad para los/as emprendedores/as, quienes, habitualmente, no cuentan con la formación ni con el conocimiento necesario en materia empresarial. Este hecho es atribuible, en líneas generales, a todo tipo de emprendedores/as, pero los actores entrevistados señalan que se agudiza en el caso de los/as de base tecnológica, ya que, a menudo, proceden de disciplinas de carácter técnico o científico en las que la formación en este tipo de materias es escasa o nula.

En este sentido, ofrecer apoyos específicos a los/as emprendedores/as en los inicios de sus iniciativas empresariales podría mejorar la presencia de la ES en el emprendimiento de base tecnológica, en la medida en la que la atención de estas necesidades puede ser una fuente de oportunidades a la hora de facilitar y resolver con éxito la creación de iniciativas. 
Así, desde el punto de vista de los actores entrevistados, en la fase de constitución de las iniciativas empresariales sería conveniente realizar actuaciones de acompañamiento individualizado, dirigidas a proporcionar la información y asesoramiento demandados por los/as emprendedores/as en relación con varios ámbitos pero, principalmente, centradas en la elaboración y firma de estatutos; en la información sobre el funcionamiento a nivel organizativo de las empresas de la ES; en relación con la idea de negocio y el plan de empresa y relativas a la provisión de información sobre instrumentos y ayudas (públicas y/o privadas) existentes.

\subsubsection{Apoyo en las fases de crecimiento y consolidación}

Para finalizar, dos ámbitos temáticos concretos han sido identificados por los actores entrevistados como especialmente relevantes para realizar actuaciones dirigidas a mejorar y fortalecer el crecimiento, la consolidación y los resultados de las empresas de base tecnológica en la ES. Estos dos ámbitos son la financiación y la cooperación.

Con respecto al primero, parece existir consenso a la hora de identificar las importantes necesidades de financiación ajena que las iniciativas de base tecnológica suelen tener en sus períodos de crecimiento y expansión como la principal barrera y freno a la creación de este tipo de empresas bajo formas jurídicas de la ES.

No obstante, es importante recordar el comportamiento diferencial de estas iniciativas en relación con los períodos de desarrollo de sus productos, que son mucho más prolongados, lo que conlleva una ausencia de ingresos suficientes por períodos de tiempo relativamente largos $y$, consiguientemente, necesidades de financiación ajena, tanto para abordar nuevas inversiones como para el circulante. En esta fase inicial, este tipo de emprendedores/as encuentra frecuentemente dificultades muy importantes para acceder a la financiación bancaria, tanto por lo novedoso de sus modelos de negocio, difíciles de encajar en los protocolos de "valoración bancaria", como por la ausencia de patrimonio inmobiliario en sus empresas, lo que desemboca en la solicitud de garantías personales 0 avales.

Por tanto, antes de profundizar en las necesidades de financiación en los períodos de crecimiento y expansión empresarial, es importante resaltar estas diferencias en las necesidades financieras y en las formas de acceso a capital de las empresas de base tecnológica dependiendo del momento de su ciclo vital, ya que las actuaciones a realizar van a ser también diferentes.

Así, durante la fase semilla, la mayoría de las necesidades de financiación ajena no bancaria son cubiertas, fundamentalmente, recurriendo a las tres FFF ("Family, Friends and Fools") y al sector público

(sobre todo, mediante programas de la Unión Europea o de organismos nacionales especializados). Por tanto, en esta fase, sería conveniente incrementar la presencia de la ES en este tipo de progra- 


\section{LA ECONOMÍA SOCIAL EN EL EMPRENDIMIENTO DE BASE TECNOLÓGICA EN ESPAÑA. UN ANÁLISIS CUALITATIVO}

mas, no solamente como fuente de financiación sino también en relación con las múltiples oportunidades que la participación en proyectos europeos puede generar.

No obstante, los principales obstáculos detectados por los actores entrevistados no se producen en la fase inicial de la actividad empresarial sino en las posteriores, de crecimiento y expansión, donde las necesidades son aún mayores, motivando que frecuentemente se necesite la entrada en la iniciativa empresarial de financiación no bancaria, bien sea mediante capital riesgo, business angels, inversores industriales u otro tipo de financiadores externos.

Y es en relación con esta entrada de financiación ajena, especialmente con la necesidad de capital riesgo, donde las personas entrevistadas señalan, de forma unánime, las mayores dificultades encontradas por las formas jurídicas de la ES, fundamentalmente vinculadas al interés de los fondos de capital riesgo por mantener el control en la toma de decisiones; las posibles dificultades percibidas en el retorno de la inversión realizada o en la venta de capital; o la escasez de experiencias por parte de este tipo de financiadores con entidades de la ES.

En este sentido, y aunque el sector ya ha comenzado a identificar actuaciones y aportar soluciones para solventar esta situación, los actores entrevistados proponen algunas medidas específicas de cara a mejorar el acceso a financiación ajena de las empresas y entidades de la ES:

- Búsqueda y contacto con inversores de perfiles interesados en financiar iniciativas con impacto social, escasos aún en España, pero presentes en otros países.

- Búsqueda de socios industriales que puedan tener interés específico en este tipo de proyectos.

- Valoración de otras fuentes alternativas de financiación como, por ejemplo, el crowdfunding y/o el crowdlending.

- Valoración de la posibilidad de introducir préstamos participativos como figura flexible de financiación.

- Valoración de la posibilidad de crear instrumentos específicos de financiación por y para el sector.

Para finalizar, y con respecto al segundo ámbito de actuación identificado, la cooperación, se destaca la importancia y necesidad de fomentar y reforzar la creación de sinergias entre los propios integrantes de base tecnológica de la ES, así como con otros sectores, como un elemento beneficioso, a corto plazo, para las iniciativas de la ES que, a medio y largo plazo, podría ser también favorable de cara a promover proyectos de cooperación de cierta envergadura, tanto por la capacidad de movilización y de difusión que la creación de estas redes conlleva como de cara a favorecer oportunidades de inversión que paliaran parcialmente las necesidades continúas de financiación de este tipo de empresas.

Así, se apunta a la necesidad de crear puntos de encuentro que favorezcan, de forma espontánea, la cooperación como un impulso institucional que ayudase a catalizar estos procesos, incre- 
mentando la cooperación entre entidades de la ES e impulsando la creación de redes con actores clave. En este sentido, el papel que desempeñan los parques tecnológicos o los viveros e incubadoras de empresas como impulsores de las transferencias de tecnologías o de la innovación, tienen un papel fundamental en la consolidación y futuro de las EBT (De Pablo Valenciano y Uribe, 2009).

\section{Discusión y potenciales líneas de trabajo}

Más allá de las actividades nucleares de las TIC analizadas en los epígrafes previos, cabe preguntarse qué posición puede ocupar la ES española en el desarrollo de los sectores emergentes, la mayoría de ellos fruto de la fusión entre las TIC y distintas actividades, qué elementos propios le pueden otorgar una posición de ventaja y qué retos deberá afrontar para aprovechar el potencial de los mismos.

Comenzando por las potencialidades, la ES en España presenta una serie de características que la sitúan en una posición de ventaja en el desarrollo esperado de las nuevas actividades. El primero de estos elementos es la fuerte especialización productiva que la ES y las entidades no lucrativas tienen en muchas de las actividades emergentes. Las empresas de la ES (incluidas asociaciones, fundaciones y otras entidades no lucrativas) representan el $8,9 \%$ en el total de empresas del tejido productivo español (DIRCE, 2017). Sin embargo, en las actividades de servicios de cuidados su presencia es muy alta: el $50 \%$ de las empresas de servicios sociales sin alojamiento y el $37,1 \%$ de las de servicios en establecimientos residenciales pertenece a la ES. Su presencia es también muy significativa en las actividades culturales $(46,8 \%)$ y en las deportivas y recreativas $(28,0 \%)$ y en parte de las actividades de la economía verde.

Un segundo elemento que sitúa a la ES en una posición aventajada es que la innovación social y la innovación organizativa, dos de los procesos clave en el nuevo escenario, son elementos identitarios de la ES. En relación con la primera, la innovación para un crecimiento más inclusivo ha sido un elemento transversal en el desarrollo de muchas entidades de la ES desde hace décadas (empresas de inserción, centros especiales de empleo, cooperativas de iniciativa social...). La innovación organizativa, que atendiendo al Manual de Oslo conlleva la introducción, entre otras, de nuevas prácticas empresariales en la organización del trabajo o de nuevos métodos para un mejor reparto de responsabilidades y de la toma de decisiones, es otro elemento nuclear de la ES y del funcionamiento de sus empresas y entidades.

De hecho, los modelos de organización de la ES podrían dar cabida en mayor medida a las preferencias de los trabajadores actuales, que demandan modelos de organización del trabajo más par- 


\section{LA ECONOMÍA SOCIAL EN EL EMPRENDIMIENTO DE BASE TECNOLÓGICA EN ESPAÑA. UN ANÁLISIS CUALITATIVO}

ticipativos, más horizontales, más inclusivos y más flexibles. Además, estos entornos son también más adecuados para el desarrollo de iniciativas de innovación social.

Por último, otro elemento a favor de que la ES sea capaz de aprovechar las oportunidades de los nuevos sectores emergentes es que su contribución económica y social está cada vez más reconocida a nivel político, social y empírico. A nivel político, la misión de las empresas de la ES está completamente alineada con los Objetivos de la Estrategia de Europa 2020 y la Agenda de la UE para el crecimiento y el empleo. De hecho, en esta última se señala que "Las empresas de la ES desarrollan aproximaciones y enfoques innovadores para dar respuesta a los cambios sociales y económicos y todo ello a través de modelos de emprendimiento que promueven la inclusión social, el respeto al medio ambiente y, en definitiva, un desarrollo económico sostenible".

A nivel empírico, recientes estudios constatan su contribución al bienestar colectivo a través de la mejora de la cohesión social y de la cohesión territorial. La valoración económica de sus intangibles está contribuyendo a visibilizar la aportación de la ES al crecimiento inclusivo. En una cuantificación reciente, se estima que los valores de las empresas y entidades de la ES aportan más de $4.000 \mathrm{M}$ euros anuales, en términos de PIB, a la economía española (Martínez et al., 2013).

Sin embargo, la posible posición de ventaja señalada en los párrafos previos puede verse lastrada por la no asunción de determinados retos. El primero de ellos es el avance hacia una mayor digitalización del sector, que debe contemplar toda la cadena de valor, desde la prestación de los servicios hasta la relación continua con el usuario (ex ante y ex post). La ES no puede renunciar a la transformación digital de sus empresas y entidades ni a desplegar todo el impacto potencial de esta transformación. El riesgo de no abordar de forma decidida los procesos de digitalización puede ser perder la posición de liderazgo actual que tiene en varios sectores (educación, servicios sanitarios, servicios sociales, agroalimentario, etc.).

Un segundo reto se centra en el ámbito de la prospección de nuevos productos y soluciones, en línea también con los métodos de la innovación social. Una de las potenciales líneas de trabajo conjunto del sector podría ser abordar sistemas de prospección y de experimentación de nuevos productos y soluciones haciendo uso de la digitalización. Un centro de prospectiva estratégica y el desarrollo de nuevas herramientas, como los "living labs", podrían tener un papel catalizador en la incorporación de la innovación social al sector. 


\section{Bibliografía}

BAKAIKOA, B., BEGIRISTAIN, A., ERRASTI, A. \& GOIKOETXEA, G. (2004): "Redes e innovación cooperativa", CIRIEC-España, Revista de Economía Pública, Social y Cooperativa, 49, 263-294.

BRUQUE, S. \& MOYANO, J. (2007): "Organisational determinants of information technology adoption and implementation in SMEs: The case of family and cooperative firms", Technovation, 27 (5), 241253. DOI: 10.1016/j.technovation.2006.12.003.

BRUQUE, S., MOYANO, J., VARGAS, A. \& HERNÁNDEZ, M.J. (2003): "Ownership Structure, Technological Endowment and Competitive Advantage: Do Democracy and Business Fit?", Technology Analysis \& Strategic Management, 15(1), 65-79.

DOI: http://dx.doi.org/10.1080/0953732032000046051.

CASTRO, R.B., SANTERO, R., MARTÍNEZ, M.I. \& GUILLÓ, N. (2013): "Impacto socioeconómico de las empresas de Economía Social en España. Una valoración cuantitativa de sus efectos sobre la cohesión social", CIRIEC-España, Revista de Economía Pública, Social y Cooperativa, 79, 35-58.

CHAVES, R. \& MONZÓN, J.L. (2012): La economía social en la Unión Europea, Bruselas: Consejo Económico y Social Europeo.

CODURAS, A. (2009): La empresa de Base Tecnológica en España: estudio de situación, Zaragoza: Fundación Zaragoza Ciudad del Conocimiento e IE Business School.

COMISIÓN EUROPEA (2002): High-Tech SMEs in Europe, Observatory of European SMEs 2002/6. Luxembourg: European Network for SME Research.

CUADRADO, M. \& CIRUELA, A.M. (2015): "Las Sociedades Cooperativas y Laborales como artífices del emprendimiento empresarial. Análisis comparativo del Perfil del emprendedor de ambas figuras en el contexto de Andalucía", CIRIEC-España, Revista de Economía Pública, Social y Cooperativa, 84, 5-34. DOI: https://doi.org/10.7203/CIRIEC-E.84.13397.

DE PABLO VALENCIANO, J. \& URIBE TORIL, J. (2009): "Emprendimiento de la economía social y desarrollo local: la promoción de incubadoras de empresas de ES en Andalucía", CIRIEC-España, Revista de Economía Pública, Social y Cooperativa, 64, 5-33.

DEL ÁGUILA, A.R. \& PADILLA, A. (2010): "Factores determinantes de la innovación en empresas de economía social. La importancia de la formación y de la actitud estratégica", CIRIEC-España, Revista de Economía Pública, Social y Cooperativa, 67, 129-155. 
ESCUELA DE ORGANIZACIÓN INDUSTRIAL (2010): Análisis de la Situación Competitiva de las Empresas de Base Tecnológica Españolas, Madrid: Fundación EOI.

ESPASANDÍN, F., CASANUEVA, C. \& GANAZA, J.D. (2004): "Las nuevas tecnologías de la información y la comunicación en la economía social andaluza", CIRIEC-España, Revista de Economía Pública, Social y Cooperativa, 49, 193-215.

FERNÁNDEZ, M.V. \& PEÑA, I. (2009): "Estrategia de innovación como factor determinante del éxito de las cooperativas vitivinícolas de Castilla-La Mancha", REVESCO, Revista de Estudios Cooperativos, 98, 70-96.

GARGALLO, A. \& PÉREZ, J. (2009): "El papel de las tecnologías de la información y la comunicación en las empresas de economía social", REVESCO, Revista de Estudios Cooperativos, 97, 90116.

GOLAFASHANI, N. (2003): "Understanding Reliability and Validity in Qualitative Research", The Qualitative Report, 8(4), 597-606. Descargado de: http://nsuworks.nova.edu/tqr/vol8/iss4/6.

HSIEH, H.F. \& SHANNON, S.E. (2005): "Three Approaches to Qualitative Content Analysis", Qualitative health research, 15(9), 1277-1288. DOI: https://doi.org/10.1177/1049732305276687.

MARTÍNEZ, M.I., CASTRO, R.B., ALEMÁN, D., GUILLÓ, N. \& SANTERO, R. (2013): El impacto socioeconómico en las entidades de economía social, Madrid: EOI, Escuela de Organización Industrial.

MARTÍNEZ, M.I., GUILLÓ, N. \& SANTERO, R. (2016): Fomento de la presencia de la Economía Social en el emprendimiento de base tecnológica y otras actividades emergentes, Madrid: CEPES Publicaciones.

MARTÍNEZ, M.I., GUILLÓ, N., SANTERO, R. \& CASTRO, R. (2017): "El impacto socioeconómico en las entidades de economía social". En: El futuro del trabajo que queremos, Madrid: OIT y Ministerio de Empleo y Seguridad Social.

MONFARDINI, E. (Dir.) (2012): "Emerging industries": report on the methodology for their classification and on the most active, significant and relevant new emerging industrial sector, Luxemburgo: PWC.

ORTíN, P., SALAS, V., TRUJILLO, M.V., VENDRELL, F. \& DE VASILIS MYRTHIANOS, C. (2007): El spin-off universitario en España como modelo de creación de empresas intensivas en tecnología, Madrid: DGPYME.

RODRÍGUEZ, G., GIL, J. \& GARCÍA, E. (1996): Metodología de la investigación cualitativa, Granada: Ediciones Aljibe.

ROSENGREN, K.E. (1981): "Advances in Scandinavia content analysis: An introduction". In: K.E. Rosengren (Ed.), Advances in content analysis, Beverly Hills, CA: Sage, 9-19. 
SALINAS, F. \& OSORIO, L. (2012): "Emprendimiento y Economía Social, oportunidades y efectos en una sociedad en transformación", CIRIEC-España, Revista de Economía Pública, Social y Cooperativa, 75, 128-151.

SÁNCHEZ, L.A. \& PÉREZ, E. (2015): "Las entidades de economía social como protagonistas de un nuevo modelo de emprendimiento y medidas legales de apoyo al emprendimiento", CIRIECEspaña, Revista de Economía Pública, Social y Cooperativa, 84, 35-62.

DOI: https://doi.org/10.7203/CIRIEC-E.84.13398.

SÁNCHEZ-ARCHIDONA, G. (2017): "Reflexiones sobre los efectos de la globalización económica en los entes de economía social. Especial referencia a la novedad de las cooperativas digitales", Lex Social, 7 (2), 250-271.

SEGUII-MAS, E., SARRIÓN-VIÑES, F., TORMO-CARBÓ, G. \& OLTRA, V. (2013): "Estudio del emprendimiento académico bajo fórmulas de economía social: análisis de las spin-off universitarias cooperativas", CIRIEC-España, Revista de Economía Pública, Social y Cooperativa, 78, 101-12.

STOREY, D.J. \& TETHER, B.S. (1998): "New technology-based firms in the European Union: an introduction", Research Policy, (26) 9, 933-946. DOI: http://dx.doi.org/10.1016/S0048-7333(97)000528.

TAYLOR, S.J. \& BOGDAN, R. (1992): Introducción a los métodos cualitativos en investigación. La búsqueda de los significados, Barcelona: Editorial Paidós.

TRENADO, M. \& HUERGO, E. (2007): "Nuevas empresas de base tecnológica: una revisión de la literatura reciente", Documento de trabajo 03, CDTI.

VARGAS, A. (2004): "Empresas cooperativas, ventaja competitiva y tecnologías de la información", CIRIEC-España, Revista de Economía Pública, Social y Cooperativa, 49, 13-29.

VARGAS, C. (2012): "Las Spin off académicas y su posible configuración como empresas de Economía Social", REVESCO, Revista de Estudios Cooperativos, 107, 186-205. 